\title{
Discours
}

Revue de linguistique, psycholinguistique et

informatique. A journal of linguistics, psycholinguistics and computational linguistics

$23 \mid 2018$

Varia

\section{Les marqueurs de changement de topique du discours en roumain : évolution sémantique et rôle pragmatique}

Alice Ionescu et Cecilia-Mihaela Popescu

\section{OpenEdition \\ Journals}

Édition électronique

URL : http://journals.openedition.org/discours/9891

DOI : 10.4000/discours.9891

ISSN : 1963-1723

Éditeur :

Laboratoire LATTICE, Presses universitaires de Caen

\section{Référence électronique}

Alice Ionescu et Cecilia-Mihaela Popescu, «Les marqueurs de changement de topique du discours en roumain : évolution sémantique et rôle pragmatique », Discours [En ligne], 23 | 2018, mis en ligne le 21 décembre 2018, consulté le 13 juin 2019. URL : http://journals.openedition.org/discours/9891 ; DOI $10.4000 /$ discours. 9891 

Revue de linguistique, psycholinguistique et informatique

\section{Les marqueurs de changement de topique du discours en roumain : évolution sémantique et rôle pragmatique}

Alice lonescu

Université de Craiova

Cecilia-Mihaela Popescu

Université de Craiova 



\title{
Les marqueurs de changement de topique du discours en roumain : évolution sémantique et rôle pragmatique
}

\author{
Alice lonescu \\ Université de Craiova \\ Cecilia-Mihaela Popescu \\ Université de Craiova
}

Cet article vise à avancer des hypothèses sur le parcours de pragmaticalisation des adverbes et des locutions adverbiales de manière et de lieu qui peuvent fonctionner comme des marqueurs de changement de topique du discours en roumain contemporain, ainsi qu'à esquisser une possible typologie de ces marqueurs. Nous avons retenu pour l'analyse les unités suivantes qui peuvent fonctionner comme des marqueurs de changement de topique du discours: altfel («sinon», «par ailleurs»), de altfel («d'ailleurs»), de asemenea ("d'ailleurs», «en outre»), (de) altminteri («par ailleurs», «d'ailleurs»), pe de altă parte («d'un autre côté», "d'un autre point de vue»), în altă ordine de idei («par ailleurs») et apropo («à propos»). Comme la plupart des marques pragmatiques dans les langues romanes, ces unités sont polyvalentes, pouvant combiner plusieurs fonctions discursives et modales au niveau du discours. Elles contribuent à l'orientation du topique de discours, ayant en même temps une valeur argumentative. Notre analyse prendra comme point de départ l'étymologie des marqueurs susmentionnés et leur évolution sémantico-fonctionnelle, pour tenter d'expliquer l'apparition, dans certains contextes d'emploi, de leur valeur pragmatique de marqueurs de changement de topique de discours (c'est-à-dire leur pragmaticalisation). Nos arguments seront illustrés par des exemples tirés d'un corpus formé de textes littéraires (fournis par les bases de données textuelles du roumain Archeus.ro, CORV [Corpus de română vorbită] et IVLRA [Interacțiunea verbală în limba română actuală]) aussi bien que d'échantillons de langue écrite et parlée prélevés sur le web à l'aide du moteur de recherche Google ou des dictionnaires en ligne Reverso et Linguee.

Mots clés: marqueurs, adverbes, topique, discours, pragmaticalisation, langue roumaine

This paper attempts to describe how adverbs and adverbial expressions of manner and space came to function as discourse markers of topic shifting in contemporary Romanian and to sketch a possible typology of these markers. We have analyzed the following units, which can function as topic shift markers: altfel ("otherwise"), de altfel ("besides"), de asemenea ("moreover"), (de) altminteri ("otherwise", "moreover"), pe de altă parte ("on the other hand", "from another point of view"), în altă ordine de idei ("on a different note") and apropo ("by the way"). Like most pragmatic markers in the Romance languages, these units are polyfunctional, as they are able to combine several discursive and modal functions at the discourse level. They contribute to the orientation of the discourse topic, while expressing an argumentative stance. Ou analysis will take the etymology of the aforementioned markers and their semantic and pragmatic evolution as a starting point, in order to explain the appearance, in certain contexts, of their pragmatic value of markers of topic shifting (i.e., their pragmaticalisation). Our arguments will be illustrated by examples drawn from a corpus of literary texts (provided by the textual databases of Romanian 
Archeus.ro, CORV [Corpus de română vorbită] and IVLRA [Interacțiunea verbală în limba română actuală]) as well as written and spoken language samples taken from the web using the Google search engine or Reverso and Linguee online dictionaries.

Keywords: pragmatic markers, adverbs, topic, discourse, grammaticalisation, Romanian

\section{Introduction}

Dans cette contribution nous nous proposons d'étudier l'évolution sémanticofonctionnelle des marqueurs discursifs (MD) de changement de topique du discours en roumain, à partir de leur origine étymologique et morphosyntaxique, pour expliquer comment ils ont pu acquérir, dans certains contextes d'emploi, des valeurs (méta)discursives.

Nos arguments seront illustrés par des exemples tirés d'un corpus formé de textes littéraires, aussi bien que d'échantillons de langue écrite et parlée, mentionnés dans la section bibliographique (Archeus.ro, CORV [Corpus de română vorbită], IVLRA [Interacţiunea verbală în limba română actuală]) ou bien prélevés sur le web à l'aide du moteur de recherche Google. Ainsi, nous avons relevé les unités suivantes, qui peuvent fonctionner dans certains contextes comme des MD de changement de topique du discours: altfel («par ailleurs»), de altfel («d'ailleurs»), de asemenea («d'ailleurs», «en outre»), (de) altminteri («par ailleurs», «d'ailleurs»), pe de altă parte («d'un autre côté», «d'un autre point de vue»), în altă ordine de idei («par ailleurs») et apropo («à propos»). Comme la plupart des MD dans les langues romanes, ces unités - à l'origine des adverbes et des locutions adverbiales de manière ou de lieu - sont polyvalentes, pouvant combiner plusieurs fonctions discursives et modales au niveau du discours. Elles contribuent à l'orientation du topique discursif, tout en ayant une valeur argumentative.

En ce qui concerne les étapes de notre étude, la première partie vise à esquisser la typologie et les parcours de pragmaticalisation ${ }^{1}$ des adverbes et des locutions adverbiales de manière et de lieu qui peuvent signaler le changement de topique du discours en roumain, tandis que la deuxième partie est dédiée à l'interprétation des données issues de la description des processus de pragmaticalisation des unités analysées dans la première section, afin de vérifier si leur parcours suit le schéma classique de l'évolution des adverbes de manière et de lieu vers le statut de marqueurs de changement de topique ou de digression.

\section{Description du cadre théorique}

Il faut préciser d'emblée que, dans la linguistique roumaine, il n'y a pas d'études consacrées spécifiquement au comportement fonctionnel et discursif des MD de

1. Le terme de «pragmaticalisation» est utilisé par Dostie (2004) pour désigner l'acquisition par divers items lexicaux ou grammaticaux d'une fonction discursive. Le terme met l'accent sur le fait que ces items ont, au niveau du discours, d'importantes fonctions pragmatiques. 
changement de topique du discours, ou au processus de pragmaticalisation dont ces unités sont issues. Quelques données sporadiques se trouvent çà et là, dans nombre d'articles (Chiorean, 2013 et 2015; Chircu, 2012; Croitor, 2015; Mîrzea Vasile, 2012), quelques monographies (Ștefănescu, 2007a et b), ou dans les dernières éditions de la Gramatica limbii române [Grammaire actuelle de la langue roumaine (GALR)] de l'Académie roumaine, mais il n'existe pas, à notre connaissance, de tentative de traitement unitaire et exclusif des marqueurs de changement de topique du discours en roumain.

Le cadre théorique général de description du fonctionnement des MD est fourni par les recherches consacrées aux marqueurs du discours dans l'espace anglophone (Traugott, 1982 et 1995; Fraser, 1996 et 2009; Lenk, 1998; Hansen et Rossari, 2005; Pons Bordería et Arguedas, 2009; entre autres) et francophone (Ducrot et al., 1980; Combettes, 2007; Charolles, 1995 et 2003; Dostie, 2004; Paillard, 2011; Fagard et Charolles, 2018; entre autres). Ces études, issues d'horizons théoriques et méthodologiques très différents, mettent en évidence l'extrême hétérogénéité des unités de la classe; en effet, comme le souligne Paillard (2011: 13), les MD «ne constituent pas un objet empirique facilement identifiable sur la base de critères qui pourraient, de façon (plus ou moins) consensuelle, être définis de façon opératoire». La polyfonctionnalité des MD est un autre facteur qui brouille les pistes et qui rend difficile un classement basé sur des critères sémantiques.

Il nous semble pertinent d'appuyer notre démarche sur la perspective proposée par Traugott qui identifie trois étapes jalonnant le processus de grammaticalisation ${ }^{2}$ (Traugott, 1982), car les expressions évoluent du statut intraprédicatif au statut de connecteur, puis à celui de modalisateur; le passage s'opère ainsi du niveau phrastique au niveau textuel, puis au niveau pragmatico-énonciatif, déterminant la pragmaticalisation de l'unité en question. Si une telle progression a été illustrée par l'analyse de certains connecteurs ou de certains modalisateurs (Schwenter et Traugott, 2000), l'étude des topicalisateurs est moins développée (voir cependant Combettes, 2003; Combettes et Prévost, 2001; Bazzanella, 1994). Le cadre évoqué a l'avantage de permettre la prise en compte, de façon cohérente, de l'opération de réanalyse: le changement de niveau de fonctionnement s'accompagne en effet d'une réinterprétation de l'énoncé, tant au niveau syntaxique qu'au niveau sémantique. Parfois l'interprétation nouvelle n'élimine pas définitivement l'ancienne, qui peut se maintenir parallèlement. C'est la généralisation de l'emploi comme marqueur de thématisation ou de topicalisation, dans des contextes nouveaux, où la construction n'aurait pas été possible auparavant, qui sera l'indice de la grammaticalisation, avec la perte de caractéristiques sémantiques, la démotivation et le figement de la locution (Sweetser, 1990; Traugott et Dasher, 2002). Les nouveaux sens se fixent sur les mots qui les véhiculent, par le biais d'un principe

2. «Grammaticalization is the process whereby lexical material in bighly constrained pragmatic and morphosyntactic contexts becomes grammatical, in other words that lexical material in specifiable syntactic functions comes to participate in the structural texture of the language» (Traugott, 1995: 1). 
général, celui de la conventionnalisation des inférences, principe décrit aussi dans Traugott et Dasher (2002). À force d'intervenir dans des contextes permettant de véhiculer une inférence enrichissant le sens initial d'un mot, ce sens a tendance à se greffer sur le mot en s'ajoutant au sens primaire. En outre, cette tendance vers la subjectivation qui motive le processus de pragmaticalisation des unités analysées, est doublée par la tendance à l'isomorphisme spécifique des langues romanes, comme nous le verrons dans la suite.

Nous considérons les MD, en suivant Paillard et Franckel (2008) et Paillard (2011), comme des unités de la langue qui ont une sémantique et une distribution spécifiques. Le propre d'un MD est, selon ces auteurs, d'être partie intégrante de la construction de la valeur référentielle d'un énoncé et de constituer un mode de dire sur le monde. Dans la perspective de l'analyse du discours (voir Reboul et Moeschler, 1998: 90), les connecteurs et les MD permettent d'accéder à la représentation de la structure du discours. Expressions à contenu procédural (Reboul et Moeschler, 1998: 75), ces unités n'encodent pas des concepts, mais des procédures. Elles nous donnent des instructions sur la manière d'accéder aux représentations mentales du locuteur (ou bien nous permettent de traiter des représentations mentales disponibles). L'ensemble de ces indices signale l'opération même de mise en mots, indique la trame du discours ou ses ruptures.

En ce qui concerne le changement de topique du discours, qui peut se réaliser tant au niveau intradiscursif qu'au niveau interdiscursif, il faut préciser que cette opération discursive peut se décliner en plusieurs nuances comme: l'introduction d'un nouveau topique, la reprise d'un topique abandonné antérieurement, une digression temporaire ou le glissement vers des topiques secondaires, dérivés, etc. Chacun de ces cas de figure nécessite l'accomplissement de certaines «conditions de réussite». Par exemple, l'introduction d'un nouveau topique est conditionnée par les connaissances du monde extralinguistique que les interlocuteurs partagent ou par leur degré de connaissance mutuelle. En fonction de la nature de la situation de communication, toutes ces opérations cognitives et discursives peuvent se réaliser de manière implicite, sans annonce verbale ou paraverbale préalable, ou bien, le plus souvent, elles se réalisent par des marquages lexicaux et/ou paraverbaux et/ou non verbaux (voir aussi GALR: 811). De ce point de vue, il faut préciser que le même lexème peut avoir plusieurs valeurs discursives amalgamées. Par exemple, en roumain, l'adverbe apropo peut exprimer l'abandon d'un topique, l'introduction d'un nouveau topique, aussi bien que l'éloignement temporaire d'un topique récemment ouvert.

\section{Hypothèses et méthodologie de la recherche}

Notre recherche, la première à traiter de la pragmaticalisation des adverbes et des locutions adverbiales de manière et de lieu à même de signaler, en roumain contemporain, le changement de topique du discours, vise à esquisser une typologie fonctionnelle de ceux-ci et à vérifier si leur parcours suit le schéma classique de l'évolution des adverbes de constituant vers le statut de marqueurs de discours. 
Nous expliquons, pour chaque unité faisant partie de la sous-classe des marqueurs de changement de topique du discours, à partir de son étymologie et de son sens primaire, les évolutions sémantiques qui se sont produites suite à son utilisation dans des contextes nouveaux, qui a motivé sa grammaticalisation et sa pragmaticalisation ultérieures.

Nos arguments seront illustrés par des exemples diachroniques tirés d'un corpus formé de textes littéraires, aussi bien que d'échantillons de la langue écrite et parlée tirés des bases Archeus.ro, CORV et IVLRA, ou bien prélevés sur le web, à l'aide des dictionnaires en ligne Reverso et Linguee et du moteur de recherche Google.

Dans ce qui suit, nous nous attacherons à décrire les valeurs et les rôles pragmatiques de chacun des MD qui actualisent le changement de topique en roumain. Mais avant d'entamer le sujet, signalons la fréquence absolue de ces lexèmes en roumain actuel, telle qu'elle résulte du schéma ci-dessous, obtenu à l'aide du moteur de recherche Google ${ }^{3}$ :

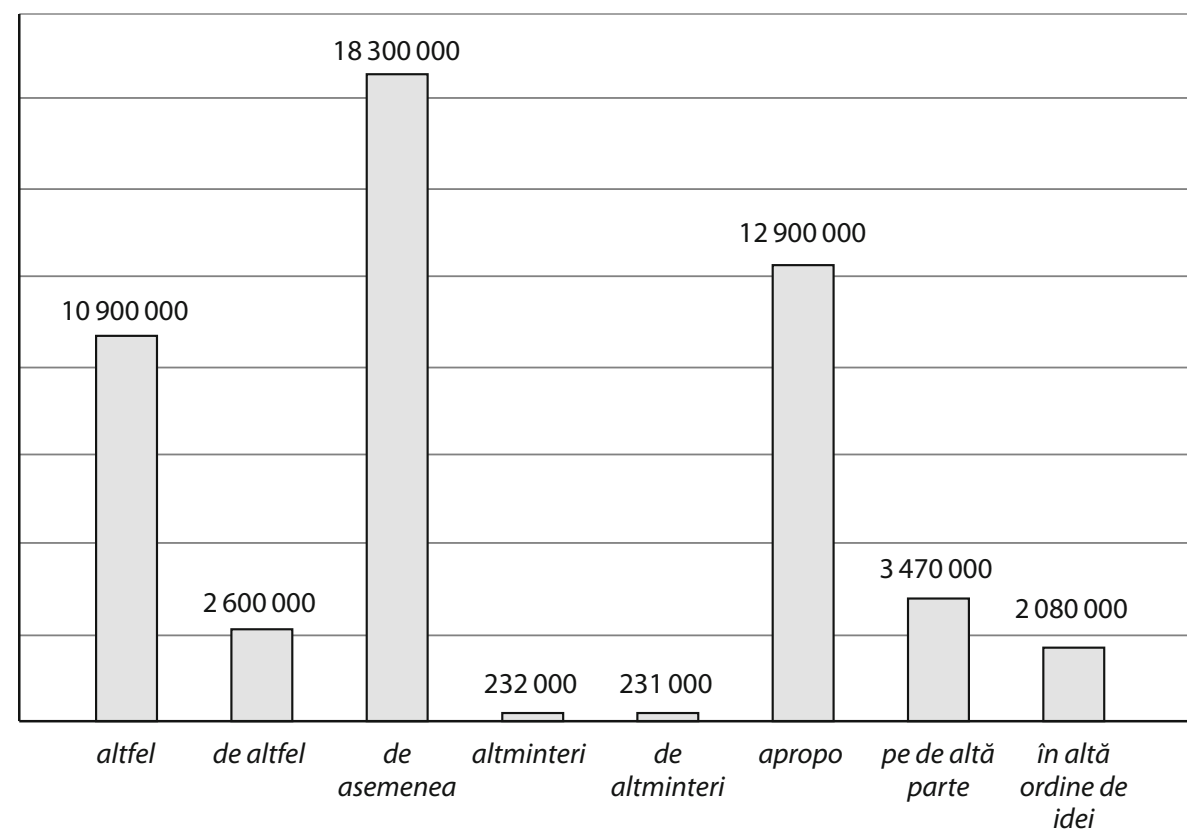

Figure 1 - Fréquence des lexèmes qui peuvent fonctionner comme marqueurs discursifs de changement de topique en roumain actuel

3. Cette statistique montre le nombre total d'occurrences de chaque lexème pris en compte par notre analyse en janvier 2018. 


\section{Parcours de pragmaticalisation des marqueurs discursifs de changement de topique en roumain}

Dans cette partie, nous donnons un aperçu des différents emplois des lexèmes constituant le paradigme des MD de changement de topique en roumain, afin d'expliquer l'acquisition de valeurs (méta)discursives par certaines unités lexicales, dans certains contextes d'emploi.

La polysémie des adverbes du roumain est, comme nous l'avons déjà précisé, le résultat d'un processus de grammaticalisation, puis de pragmaticalisation: une même unité lexicale peut, selon sa portée (en liaison avec sa position et sa prosodie), fonctionner tantôt comme adverbe de constituant, tantôt comme adverbe connecteur ou encore comme marqueur de changement de topique du discours (ou introducteur d'un nouveau topique).

\subsection{Altfel (litt. autre façon): «autrement», «sinon »; et de altfel (litt. d'autre façon) : «d'ailleurs», «par ailleurs»}

L'évolution de l'adverbe altfel (fr. «autrement», «d'une autre manière»), pourrait être résumée de la manière suivante:

1. adverbe de manière, déterminant du verbe, comme sous [1] ;

2. connecteur adversatif, comme en $[2 \mathrm{a}]$ et $[2 \mathrm{~b}]$;

3. marqueur de changement de topique du discours (fr. «autrement», fam. «sinon»), comme en [3].

[1] Urmează un joc important pentru noi şi pentru suporteri pentru că se ştie că meciurile cu Dinamo sunt tratate altfel.

(https://www.digisport.ro)

'Le match suivant est important pour nous, ainsi que pour nos fans, puisqu'on sait bien que les matches avec Dinamo sont traités d'une manière différente.'

(notre trad.)

[2] a. Ciocul mic, altfel te împușc! (http://www.b1.ro/stiri/eveniment)

'Ta gueule ou je te tue!' (notre trad.)

b. Altfel, de ce te-ai filma? [emploi intradiscursif]

'Autrement, pourquoi on se filmerait?'

[3] Altfel, ce mai faci? [emploi interdiscursif]

'Sinon/Autrement, comment vas-tu?'

L'adverbe roumain altfel (ayant pour variantes diachroniques ou dialectales les formes: altfeliu, alfel) est une composition récente - selon un modèle hongrois (Chircu, 2012: 58) - issue, par la grammaticalisation, du nom d'origine hongroise fel, 
«manière», agglutiné avec le prédéterminant indéfini alt, «autre» (CDER [Dicţionarul etimologic al limbii române] : s. v. «Altfel»). Il apparaît, dès ses premières occurrences dans des textes écrits au début du XVIII siècle (le mot soudé est attesté en 1703, dans le Dictionnaire de Théodor Corbea, voir RDW [Rumänisch-Deutsches Wörterbucb]), chargé d'une signification primaire adverbiale, exprimant la manière (plus précisément, l'idée d'altérité, de différence) et modifiant un nom (exemple [4]) aussi bien qu'un verbe (exemple [5]):

[4] Atuncea cînd pohtește pentru ne rebdarea păcii pentru îndoiale au pentru alte feliu [altfel] de ocă.

(Lászlo Pal Barany, Pânea Pruncilor sau Învățătură credinții creștinești, strînsă in mică şumă, Cluj-Napoca, Argonaut, 2008, 64 v , in Chircu, 2012: 58)

'Lorsqu'il cherche le conflit, lorsqu'il est en doute ou qu'il cherche un autre type de querelle.'

(notre trad.)

[5] [...] însă nici numele lor într-alt feliu să mai mutase și să mai schimbase, să le zică vlahi sau într-alt chip.

(Constantin Cantacuzino, Istoriia Țărâi Rumânești, Bucarest, Editura Academiei Române, 2006, vol. I, 56 ro p. 183, in Chircu, 2012: 57)

'[...] mais leur nom n'avait pas encore changé, on ne les appelait pas «vlahi» ou autrement.'

(notre trad.)

Dans ce type d'occurrence, altfel pourrait être remplacé par des équivalents, tels que într-alt chip comme dans l'exemple [5], $\operatorname{altcum}(v a)$, altmintrelea, c'est-àdire des adverbiaux construits sur la base du même adjectif indéfini alt. Ce type d'emploi se retrouve dans toutes les étapes de l'évolution du roumain et il continue à exister de nos jours encore. Au XIX ${ }^{\mathrm{e}}$ siècle, altfel se trouve déjà complètement grammaticalisé; il est donc ressenti comme une unité lexicale indépendante du point de vue syntaxique. Cela lui permet d'apparaitre accompagné du quantificateur adverbial mai, "plus», ou bien d'occuper une position initiale, finale ou médiane dans l'énoncé:

[6] Să-1 vedeți pe cal, veți judeca altfel.

(Costache Negruzzi, Scrieri, Bucarest, Editura Socec, 1872, vol. I, p. 40, in DLRLC

[Diç̧ionarul limbii române literare contemporane])

'Si vous le voyez à cheval, vous le percevrez autrement.'

(notre trad.)

[7] Acela era oleacă mai chipos și altfel îmbrăcat.

(Ion Creangă, Povestea lui Stan Pățitul, in Povești, Bucarest, Editura Ion Creangă, 1984, p. 148, in DLRLC) 
'Celui-là était un peu moins laid et habillé autrement.'

(notre trad.)

[8] Altfel n-ai cum să le zici.

(Ion Urban Jarník et Andreiu Bîrseanu, Cântece și strigături din Ardeal date la iveală de, Brașov, Editura Librăriei Ciurcu, 1903, p. 263, in DLRM)

'On ne peut pas le dire autrement.'

(notre trad.)

C'est vers la fin du XIX ${ }^{\mathrm{e}}$ siècle et le début du XXe que l'adverbe altfel - le plus souvent accompagné par la conjonction explicative $c \breve{a}(c i)$, «car»-commence à être retrouvé, seul ou précédé par une préposition (voir l'exemple [11] infra), dans des structures syntaxiques spécifiques où il a la fonction de connecteur adversatif, équivalant aux locutions conditionnelles dacă $n u$, de unde $n u$, în caz contrar ou à l'adverbe altmintrelea (fr. «sinon», «en cas contraire»):

[9] Noroc... că era o fată robace și răbdătoare, căci altfel ar fi fost vai ș-amar de pielea ei. (Ion Creangă, Fata babei şi fata moșului, in Poveşti, Bucarest, Editura Ion Creangă, 1984, p. 283, in DLRLC)

'Heureusement qu'elle était une fille travailleuse et patiente, car autrement elle aurait eu beaucoup de mal.'

(notre trad.)

[10] Trebuie să știm de unde ne tragem, căci altfel suntem niște nenorociți rătăciți.

(Alexei Mateevici, «Cuvintarea la primul congres al învăţătorilor moldoveni din Basarabia», SScoala moldovenească, vol. 1, n 3-4 [juillet-septembre], 1917, in Archeus.ro)

'Nous devons connaître nos origines, sinon nous sommes des infortunés de l'histoire.' (notre trad.)

[11] Ascunde-te, Romeo, că-ntr-altfel ești pierdut.

(Alexandru Macedonski, Opere, Bucarest, Editura Fundației Naţionale pentru Știință și Arte - Editura Univers Enciclopedic, 2008, vol. II, Dramaturgie. Traduceri și adaptări în limba română. Versuri. Proză, p. 151, in DLRLC)

'Cache-toi, Roméo, sinon tu es perdu.'

(notre trad.)

Tout en actualisant une «hypothèse négative» (voir Mongi, 2009: 148) - «p altfel q»-, altfel remplit la fonction de prophrase conditionnelle (voir Mîrzea Vasile, 2012: 39). Dans de telles occurrences - repérables, en général, à l'aide de la périphérie gauche (qui, dans la plupart des cas, est une protase conditionnelle réalisée avec un impératif ou un subjonctif hortatif) -, le lexème 
analysé (accompagné ou non d'une préposition ou de la conjonction că, «que») devient un pseudo-circonstant connecteur (voir Ștefănescu, 2007b : 29) ${ }^{4}$ qui peut figurer dans la classe des MD.

Avec cette nouvelle valeur, il se rencontre aussi dans la langue actuelle où il est devenu «un marqueur discursif spécialisé dans l'introduction des propositions métadiscursives liées à l'idée d'énonciation ou de compréhension» (Ștefănescu, 2007b : 29 - notre trad.) ${ }^{5}$.

De telles structures traduisent une certaine modalisation épistémique, actualisant une atténuation, une probabilité ou, tout simplement, l'univers de croyance du locuteur ou encore le degré de prise en charge du contenu propositionnel asserté. Par ailleurs, il faut ajouter qu'en roumain contemporain, ces moules syntaxiques, devenus de véritables «routines conversationnelles» (Ștefănescu, 2007b : 34-35), se sont élargis, dans le sens où, dans le premier segment de ce cadrage discursif, on peut trouver non seulement l'impératif (exemples [11], [12] et [13]), mais aussi un verbe modal du type a putea, «pouvoir» (exemple [14]), a trebui, «falloir» (exemple [10]), a se părea, «sembler», etc., ou bien l'indicatif présent ou passé (exemple [13]).

[12] Mănâncă, altfel nu pleci la joacă!

(Mîrzea Vasile, 2012: 39)

'Mange, sinon tu ne sortiras pas jouer!'

(notre trad.)

[13] Munciţi, că altfel vă ia mama dracului! ${ }^{6}$

(Internet, in Ștefănescu, 2007b : 29)

'Travaillez bien, sinon vous serez punis!'

(notre trad.)

4. «Aceste circumstanţiale se caracterizează printr-o slabă integrare semantico-sintactică în enunţ. Omisiunea lor din enunţ nu $\hat{\imath} l$ dezorganizează sintactic şi nu $̂ l$ fac cu mult mai sărac din punct de vedere semantic. Aceste circumstanţiale de modalitate se autoinclud, la rândul lor, în clasa circumstanţialelor metadiscursive (GALR, II, p. 468-469). Functiile sintactice care le sunt atribuite sunt de un tip special, sunt functii-satelit ale circumstanţialului de mod și ale circumstanţialelor în general, sunt funcții periferice din punct de vedere al importanţei lor sub aspect semantic» (Ștefănescu, 2007b : 29) [Ces circonstants se caractérisent par un faible degré d'intégration sémantique et syntaxique dans l'énoncé. Leur omission n'affecte pas l'organisation syntaxique de l'énoncé et ne l'appauvrit pas du point de vue sémantique. Ces circonstanciels de modalité font partie, à leur tour, de la classe des circonstanciels métadiscursifs (GALR: 468-469). Les fonctions syntaxiques qu'on leur attribue sont d'un type particulier: généralement ce sont des satellites des circonstants, ayant des fonctions périphériques comme importance sémantique - notre trad.].

5. «Sintagma conectivă că altfel a devenit un marcator discursiv specializat pentru introducerea unor propoziţii metadiscursive legate de ideea enunţării şi a înţelegerii» (Ștefănescu, 2007b : 34-35) [Le syntagme connectif că altfel est devenu un marqueur discursif spécialisé pour l'introduction des propositions métadiscursives liées à l'énonciation ou à la compréhension - notre trad.].

6. Litt. «Travaillez, sinon la mère du diable vous emportera!». 
[14] Poate că asta e vreo poantă, că altfel nu-mi explic!

(Internet, in Ștefănescu, 2007b : 29)

'Peut-être qu'il s'agit d'une plaisanterie, sinon je ne me l'explique pas!'

(notre trad.)

[15] E un idiot, altfel nu știu ce să mai zic!

(Internet)

'C'est un idiot, sinon je ne sais pas quoi dire!'

(notre trad.)

Une autre signification, enregistrée vers le milieu du XIX siècle, apparaît dans des structures semblables aux emplois présentés supra, altfel étant équivalent cette fois-ci à l'adverbe altminteri, "par ailleurs», «d'ailleurs», ou bien à la locution adverbiale in rest, «du reste». À la différence des tournures hypothétiques décrites sous [12] à [15], altfel est ici un véritable connecteur à valeur métadiscursive, employé pour assurer une certaine cohérence dans la structuration du discours et signalant plutôt une séquence digressive. De telles tournures semblent exprimer un autre point de vue du locuteur ou parfois même la voix ou la rumeur publique [+ valeur évidentielle] :

[16] Cantemir era un domn. Altfel prea de treabă om.

(Costache Negruzzi, Scrieri, Bucarest, Editura Socec, 1872, vol. I, p. 40, in DA [Diç̧ionarul limbii române] / DLR [Diç̧ionarul limbii române. Serie nouă])

'Cantemir était un grand seigneur. Autrement, c'était un homme trop gentil.'

(notre trad.)

[17] Bulgarii aceia erau oameni pacinici... Altfel, aici pace și liniște.

(Ion Luca Caragiale, Momente și schițe (Cum stăm...), Bucarest, Editura Minerva, 1983, in Archeus.ro)

'Ces Bulgares-là étaient des gens paisibles... Sinon, ici règnent la paix et la tranquillité.' (notre trad.)

[18] Şi-a muşcat, cu o satisfaç̧ie vulgară, buza de jos, ca şi când ar fi spus, câştigând prinsoarea : „A, domnule, în sfârşit!” Altfel întrevederea a fost frumoasă, cu lunecuşuri de ironie voit banală şi superficială, ca să pară tandră şi indiferentă.

(Camil Petrescu, Ultima noapte de dragoste, intâia noapte de război, Bucarest, Editura Eminescu, 1980, p. 97)

'Elle s'est mordu, avec une satisfaction vulgaire, la lèvre inférieure, comme si elle avait gagné un pari: «Ah, Monsieur, enfin!» Autrement l'entrevue a été sympa, avec des plaisanteries banales et des ironies superficielles et amicales, qui lui ont donné un air bénin.'

(notre trad.) 
Dans ce type d'occurrence, altfel est généralement détaché en tête de phrase et il est même emphatisé, étant séparé par une virgule du reste de l'énoncé. De telles structures apparaissent tant au niveau intradiscursif, comme en [19], qu'au niveau de l'échange conversationnel, où altfel peut marquer aussi un changement de topique, comme en [20].

[19] - Auzi acolo la Safta... şi ea are un copil ce se face măricel. Ast copil l-a născut patru luni în urma măritişului cu nea Ghiţă. În curând o să aiba doi, şi de, se zic cam multe... Altfel, nea Ghiţă e omul lui Dumnezeu, ce-are el cu gura lumii ? Până nu vede, nu crede... Dacă o crede ş-atunci...

(Barbu Ştefănescu Delavrancea, Sultănica, Bucarest, Editura Minerva, 1972, p. 273)

'Regarde-moi cette Safta... elle aussi, elle a un enfant qui sera bientôt grand. Cet enfant, elle l'a eu quatre mois après son mariage avec Ghiţă. Bientôt elle en aura deux, et... eh bien, le monde dit des choses à son compte... Autrement/sinon, Ghiţă est l'homme le plus gentil du monde, il s'en fout de ce que le monde parle... Il n'en croit qu'à ses yeux... et encore!'

(notre trad.)

[20] Altfel, ce mai faci? Văd că ai făcut destule de curând.

(https://www.linguee.com/)

'Et sinon tout va bien pour vous? Vous avez été bien occupé ces derniers temps.'

(https://www.linguee.com/)

D'ailleurs, comme MD de changement de topique, altfel apparaît dans quelques structures à peu près fixes, du type : altfel + question simple réalisée avec les verbes a zice, a spune («dire»), a face («faire»), a (se) auzi («entendre»), a se ocupa («s'occuper»), a lucra («travailler»), d'où le fait qu'il puisse être omis sans affecter la signification globale de l'énoncé.

Si altfel s'est spécialisé plutôt comme MD à valeur métadiscursive, sa variante de altfel («d'ailleurs»), qui résulte de la grammaticalisation complète de la préposition de et du connecteur altfel, fonctionne comme MD à valeur métadiscursive, fréquemment comme démarqueur ayant le rôle de prolonger l'échange comme dans l'exemple [25]. De plus, il sert à introduire un nouveau topique représentant un nouveau point de vue (exemple [21]), une argumentation (exemple [22]), une justification ou bien il est employé pour renforcer l'idée précédente (exemple [23]). Avec ces valeurs - développées probablement vers la fin du XIX ${ }^{e}$ siècle et le début du $\mathrm{XX}^{\mathrm{e}}$ siècle (car nous n'avons trouvé aucune occurrence chez les écrivains classiques, comme Ion Creangă ou Ion Luca Caragiale, mais, en revanche, Calistrat Hogaș, l'utilise abondamment) -, de altfel peut se placer en tête de phrase, en position médiane (exemple [24]) ou finale (exemple [23]) et même en incise (exemple [27]) :

[21] Prin rasa lui sclivisită şi lustruită de murdărie se vedeau stelele ; iar de cămaşă nu mai putea fi vorbă, fiindcă acea în care își primise darul se topise demult pe el; și știut 
este că un călugăr trebuie și să moară în cămașa în care și-a căpătat sfântul său chin. De altfel, Săhăstria numără vreo șase viețuitoare de soiul arătat mai sus; întâmplarea însă ne scosese, dintre toate, pe tipul cel mai desăvârșit.

Așadar, spre a lua iarăși firul povestirii, singuraticul călugăr culegea săcărică și, pe cât aflarăm, făcea și el o mică negustorie cu ea.

(Calistrat Hogaș, Pe drumuri de munte, Bucarest, Editura Viaţa Românească, 1912, p. 17)

'Son froc en lambeaux, poli par la crasse, laissait entrevoir les étoiles; on ne pouvait pas parler de chemise, car celle qui lui avait servi pendant son noviciat était depuis longtemps dépecée. On sait bien qu'un moine est censé expier dans la chemise avec laquelle il a vécu son saint supplice. D'ailleurs le monastère Săhăstria compte environ six âmes de cette espèce et c'est le hasard qui a fait surgir devant nous le type le plus représentatif.

Donc, pour reprendre le fil de notre histoire, le moine solitaire était en train de cueillir du brome et, comme nous allions l'apprendre tout de suite, il faisait aussi un peu de commerce avec.'

(notre trad.)

[22] [...] şi diverşii scriitori care scriu astăzi, dacă ar fi făcut aşa, ar fi fost, desigur, cu neputință să fie grupați. De altfel, un grup omogen și statornic, în afară de cei ce țin rubrici speciale, adică omogenitate anonimă, nu a fost niciodată. Scriitorii s-au perindat la întâmplare, au apărut și au dispărut.

(Dimitrie Anghel, «Represiune pentru represiune», Ramuri, VI, 5 [22], 7 novembre 1910, p. 65-67, in Archeus.ro)

'[...] et tous les autres écrivains d'aujourd'hui, s'ils avaient fait ainsi, auraient été impossibles à regrouper. D'ailleurs, il n'a jamais existé un groupe homogène et stable, excepté ceux qui tiennent une rubrique de journal, c'est-à-dire un groupe homogène mais anonyme. Les écrivains sont apparus au hasard, puis sont disparus.' (notre trad.)

[23] Lucrurile vizibile îl captivau numai ca semne de stări psihice, și în acest caz, desigur, le vedea cu o rară inteligenţă. De altfel, el mărturisește categoric că nu era vizual.

(Paul Zarifopol, Creație și analiză, Bucarest, Cartea Românească, 1930, in Archeus.ro)

'Les choses visibles le captivaient seulement en tant que signes d'états d'âme et dans ce cas-là, bien sûr, il les voyait avec une rare intelligence. D'ailleurs, il avoue qu'il n'était décidément pas un visuel.'

(notre trad.)

[24] Mă simţeam alb, cu tot sufletul în aşteptare şi liniştit ca un cadavru. Era, de altfel, un fior în întreaga încăpere, care a făcut să nu mai respire nimeni.

(Camil Petrescu, Ultima noapte de dragoste, întâia noapte de război, Bucarest, Editura Eminescu, 1980, p. 14) 
'Je me sentais tout blanc, mon âme pétrifiée par l'attente et j'étais muet comme un cadavre. Il y avait, d'ailleurs, une certaine vibration dans la chambre qui faisait que personne ne respirait plus.'

(notre trad.)

[25] Niciodată n-am fost lovit ca bărbat şi cred că n-aş putea îndura asta. De altfel, mai ajunsesem de două ori poate, în viaţa mea, până în acest prag.

(Camil Petrescu, Ultima noapte de dragoste, întâia noapte de război, Bucarest, Editura Eminescu, 1980, p. 44)

'En tant qu'homme, je n'ai jamais été giflé et je ne crois pas être capable de le supporter. D’ailleurs, j’étais arrivé seulement deux ou trois fois dans ma vie dans cette situation.' (notre trad.)

[26] Visul oricărui politician este să câștige în alegeri. Un lucru foarte normal, de altfel. (Cristian Tudor Popescu, Copiii fiarei, Iaşi, Editura Polirom, 2013 [3e éd.], p. 29)

'Le rêve de tout homme politique et de gagner les élections. Chose tout à fait normale, d'ailleurs.'

(notre trad.)

[27] Concentrarea începea să-mi joace feste (de altfel, boală veche, și în săteasca circulație din România aveam niște stopuri mentale, absențe de câteva secunde, după care taică-meu îmi îndrepta volanul strigând).

(Cristian Tudor Popescu, Copiii fiarei, Iaşi, Editura Polirom, 2013 [3 éd.], p. 53)

'Mon attention devenait défaillante (maladie ancienne, d'ailleurs, puisque dans la circulation rurale de Roumanie il m'arrivait jadis des syncopes, des moments d'absence qui duraient quelques secondes, à la suite desquelles mon père saisissait le volant en criant).'

(notre trad.)

\section{2. $\quad$ Altminteri (litt. autrement)}

Avec ses nombreuses variantes dialectales ou diachroniques - al $(t)$ mint $(e) r e(a)$, al(t)mintre(le)a, aimint(e)re(a), aimint(e)ri(lea), al(t)mintre(le)a, amintre(le) -, altminteri est un mot très ancien, ayant un parcours de grammaticalisation intéressant et un fonctionnement complexe. Il provient vraisemblablement du syntagme latin ALIA MENTE contaminé avec altera mente, une possible forme intermédiaire étant *altrăminte, qui aurait pu donner par métathèse alt $(\breve{a})$ mintre (voir aussi CDER: s. v. «Alminteri»). En tout cas, comme le remarque Pușcariu, c'est la seule formation adverbiale avec $-M E N T E$ en roumain.

Attesté pour la première fois en 1521 dans Scrisoarea lui Neaç̧u de la Câmpulung către judele Braşovului Hans Benckner, le mot fonctionnait initialement comme adverbe de manière, modifieur du verbe, et il exprimait l'altérité dans la manière. 
[28] I pak dau știre domnietale za lucrul turcilor, cum am auzit eu că împăratul au ieşit den Sofiia şi aimintrea nu e. [syn. : in alt chip, altfel, altcum, fr. «autrement»]

(Scrisoarea lui Neaç̧ de la Câmpulung [document manuscrit])

'Je veux annoncer à votre excellence les mouvements des Turcs, parce que j'ai entendu dire que l'empereur a quitté Sofia et il n'en est pas autrement.'

(notre trad.)

On le retrouve avec la même valeur dans quelques documents écrits aux XVIII et $\mathrm{XIX}^{\mathrm{e}}$ siècles:

[29] Altmintrelea îl numeşte în faţă, almintrelea pe din dos, unde vorbeşte cu îndrăzneală. (Petru Maior, Procanon, propovedanii, didacbii, istoria pentru inceputul românilor în Dachi [1783], Bucarest, Editura Minerva, 1976, in Mîrzea Vasile, 2012)

'Il s'adresse à lui d'une certaine manière et le nomme d'une autre manière derrière son dos, où il parle avec audace.'

(notre trad.)

[30] Așa gătită îi ședea și mai bine decît altminteri.

(Petre Ispirescu, Legendele sau Basmele românilor, Bucarest, Editura Socec, 1872, vol. I, p. 352, in DLR)

'Habillée ainsi elle était encore mieux qu'avant.'

(notre trad.)

Plus tard, vers la fin du XIX ${ }^{e}$ siècle, il est employé aussi comme adverbe connecteur adversatif (syn. en roum. : dacă $n u . .$. ; équivalent en fr.: «sinon»), comme dans l'exemple [31], ou bien comme adverbe modalisateur (syn. en roum. : încolo, in rest; équivalents en fr.: «autrement», «du reste»), comme dans l'exemple [32]:

[31] Fă ce-ți poruncesc eu, altminteri va fi rău de tine.

(Petre Ispirescu, Legendele sau Basmele românilor, Bucarest, Editura Socec, 1872, p. 87)

'Fais ce que je t'ordonne, sinon tu finiras mal.'

(notre trad.)

[32] El ştia să lucreze bine, dară era aşa de leneş încât tânjea pământul supt el... Altminteri era deştept şi isteț.

(Petre Ispirescu, Legendele sau Basmele românilor, Bucarest, Editura Socec, 1872, p. 132)

'Il était bon laboureur, mais il était si fainéant que ses terres étaient délaissées... Autrement il était intelligent et adroit.'

(notre trad.) 
La locution adverbiale de altminteri, formée de la préposition de et de l'adverbe altminteri, est devenue un véritable MD de changement de topique du discours (équivalant en français à d'ailleurs, par ailleurs) au XVII ${ }^{\mathrm{e}}$ siècle, mais elle est de moins en moins utilisée en roumain contemporain, probablement à cause de son phonétisme compliqué.

[33] Theodora se cunună cu Theofil. De aimintrea era om bun şi nu iubiia strâmbătăţile şi pre $\mathrm{H}$ (risto)s proslăvea.

(Mihail Moxa, Cronica universală/Cronograf [1620], Bucarest, Editura Minerva, 1989, in Croitor, 2015: 250)

'Théodora épousa Théophile. Celui-ci était, d'ailleurs, un brave homme qui n’aimait pas l'injustice et qui honorait Notre Seigneur Jésus.'

(notre trad.)

[34] Drum ca acela care trece prin Valea-Seacă înspre Sărăceni jur-împrejur nu este. [...] Pe aicea sărăceneanul se simte acasă, pentru aceea mână numai în paşi. De altminterea, nici nu i se urăşte.

(Ioan Slavici, Popa Tanda, Sibiiu, Editura Asociaţiunii, 1914, p. 38)

'Il n'y a pas de meilleure route que celle qui passe par Valea-Seacă en allant vers Sărăceni. [...] Par ici, les villageois se sentent comme chez eux et ralentissent le rythme de leurs pas. D'ailleurs, ils n'ont pas de hâte, car ils ne s'y ennuient pas.'

(notre trad.)

[35] Se spune că cei Treizeci au primit deja sute de denunțuri; de altminteri, orașul nostru a fost întotdeauna înțesat de spioni. Exemplele sunt nenumărate și însiruirea lor ar fi nespus de plicticoasă.

(http://www.reverso.net/)

'On dit que les Trente ont déjà reçu des centaines de rapports. D'un autre côté, notre ville a toujours été pleine d'espions. Nous avons quantité d'exemples à citer.'

(http://www.reverso.net/)

[36] Este, de asemenea, necesară o mai bună armonizare europeană, după caz. De altminteri, legislația nu rezolvă toate problemele. Trebuie respectată litera, dar şi spiritul directivei-cadru și ale directivelor sectoriale de către statele membre, atunci când se aplică legislația.

(https://www.linguee.com/)

'Il faut aussi faire progresser l'harmonisation européenne lorsque cela est pertinent. Par ailleurs, la législation ne fait pas tout. Il faut que la lettre et l'esprit de la directivecadre et des directives sectorielles soient pleinement respectés par les États membres lorsqu'ils procèdent à la mise en œuvre de cette législation.'

(https://www.linguee.com/) 


\subsection{De asemenea (litt. de même) : «d'ailleurs», «en outre»}

La locution de asemenea (avec les variantes diachroniques ou dialectales de asemeni, de asemine ou de asemene) est l'un des principaux connecteurs métadiscursifs en roumain actuel, qui sert à l'organisation discursive, en actualisant toute une série de rôles argumentatifs, tels que l'ajout de nouvelles informations, le changement ou le développement d'un topique.

Cette locution, issue de la composition de l'adverbe asemenea, «de la même manière» (du lat. ASSIMILE, «semblable»), et de la préposition de, est attestée pour la première fois au XVI siècle, dans Palia de la Orăştie. À cette époque-là, asemenea actualisait, précédé ou non de la préposition $d e$, la comparaison entre deux prédications, entre deux situations, comme on peut le voir en [37]:

[37] Și toți asemene vor sta, cine și cu ale sale păcate vor sta.

(Bogdan Petriceicu Hașdeu, Cuvente den bătrâni, Bucarest, Editura Cultura Națională, 1937, vol. II, p. 449, in DLR)

'Et tous de même se tiendront debout pendant le jugement, chacun avec ses péchés.' (notre trad.)

Cette comparaison pouvait être parfois apparente (le terme comparé ne figurant plus dans la construction). Ainsi, à partir du XIX ${ }^{\mathrm{e}}$ siècle, l'unité analysée commence-t-elle à exprimer l'introduction d'une nouvelle information, le plus souvent à caractère inattendu, pouvant figurer en position médiane (voir les exemples [39], [40] et [41]) ou finale dans l'énoncé.

[38] [Adunarea sau soborul a toată țara] se convocau de domn pentru facerea legilor... Ele judecau asemenea în pricinile de vini mari politice.

(Nicolae Bălcescu, Opere, Bucarest, Editura Fundației Naţionale pentru Știinţă şi Arte - Muzeul Național al Literaturii române, 2017, vol. II, p. 13, in DLRLC)

'L'assemblée était convoquée par le prince régnant pour donner des lois. Elle jugeait de même les causes de nature politique.'

(notre trad.)

[39] Tu-mi cei chiar nemurirea mea/ în schimb pe-o sărutare./ Dar voi să știi asemenea/ Cît te iubesc de tare.

(Mihai Eminescu, Opere, Bucarest, Editura Academiei Române, 1939, vol. I, p. 173, in DLRLC)

'Tu me demandes même mon immortalité en échange d'un baiser. Mais je veux que tu saches de même combien je t'aime.'

(notre trad.)

Par la suite, à partir du XIX ${ }^{\mathrm{e}}$ siècle, la locution de asemenea est devenue aussi un équivalent de pe lângă acestea («à côté de»), sii... («et»), încă («encore», «aussi»), cu acest prilej («à cette occasion»), tout en manifestant une grande mobilité d'insertion. 
[40] - Sigur - întăreşte altul - dovadă că batalionul nostru n-a fost angajat în târg la Bran; la Olt, de asemenea, a fost rezervă.

(Camil Petrescu, Ultima noapte de dragoste, întâia noapte de război, Bucarest, Editura Eminescu, 1980, p. 17)

'- Bien sûr, confirma un autre, la preuve est que notre bataillon n'a pas été engagé à Bran; à la bataille d'Olt il a été en réserve également.'

(notre trad.)

En roumain contemporain, la locution de asemenea peut remplir la fonction de MD de changement de topique seulement si elle est employée en tête de phrase, disjointe du reste de l'énoncé, pouvant être remplacée aussi par tot aşa/ tot astfel/ la fel/ în acelaşi fel («de même») ou totodată («en même temps»).

[41] De asemenea, solicitantul afirmă că înlăturarea prejudiciului se datorează în principal existenței acestor măsuri și că $[. .$.$] orice noi importuri substanțiale la prețuri de$ dumping din țările în cauză ar avea ca efect reapariția unor prejudicii pentru industria comunitară.

(https://eur-lex.europa.eu/)

'Par ailleurs, le requérant soutient que l'élimination du préjudice est due principalement à l'existence de mesures antidumping et que [...] toute reprise des importations en volumes importants et à des prix de dumping en provenance des pays concernés conduirait probablement à une réapparition du préjudice pour l'industrie communautaire.'

(notre trad.)

[42] De asemenea, Tribunalul a săvârșit o eroare atunci când a afirmat (sau a presupus în mod implicit) că articolul 55 alineatul (4) din regulament conferă Oficiului puterea deplină de apreciere [...].

(https://eur-lex.europa.eu/)

'En outre, le tribunal affirme (ou considère implicitement) à tort que l'article 55, paragraphe 4 , du règlement donne à l'OCVV un entier pouvoir d'appréciation [...].' (notre trad.)

\subsection{La locution adverbiale pe de altă parte}

La locution adverbiale pe de altă parte (litt. «sur de autre côté») - une périphrase ayant la structure: prép. $p e+$ prép. $d e+$ adj. indéf. alta $\breve{a}$ («autre») + nom parte («partie») - a acquis récemment en roumain une valeur métadiscursive (équivalant aux tournures «d'un autre côté», «en revanche», «par ailleurs», en français), pouvant introduire un nouveau topique de discours ou un point de vue nouveau sur le topique courant. Au début, cette locution avait un sens spatial, apparaissant en corrélation avec la structure pe de o parte (fr. «d'un côté»), qui la précédait: 
[43] De o parte şi de alta a râului se întind ogoare roditoare.

(https://dexonline.ro/)

'D'un côté et de l'autre de la rivière il y a des champs cultivés.'

(notre trad.)

Plus tard, cette locution a acquis un sens figuré, subjectif, exprimant une comparaison, souvent apparente. La structure pe de o parte... pe de alta/ pe de altă parte (fr. «d'un côté... de l'autre») est une locution adverbiale intégrée à la phrase qui exprime une mise en parallèle et une opposition (la grammaire du roumain appelle ce type de constructions des «locutions adverbiales à sens modal»).

[44] Pe de o parte îl înțeleg, pe de alta nu.

(notre exemple)

'D'un côté je le comprends, de l'autre, non.'

(notre trad.)

Dans les deux exemples ci-dessous, pe de altă parte apparaitt sans son antécédent pe de o parte, détaché, après un nom propre, et exprime une opposition ou une mise en parallèle de personnes ou de situations.

[45] Gilderoy Lockhart, pe de altă parte, era imaculat, în nişte veşminte dărâmătoare, turcoaz, iar părul auriu îi strălucea pe sub pălăria cu ornamente aurii.

(ParaSol)

'Gilderoy Lockhart, en revanche, était impeccable dans sa robe de sorcier turquoise, avec ses cheveux dorés qui brillaient sous un chapeau également turquoise, bordé de fils d'or.'

(ParaSol)

[46] Ron, pe de altă parte, părea foarte fericit, cum nu mai fusese de mult timp.

(ParaSol)

'Il y avait longtemps, en revanche, que Ron n'avait pas paru aussi heureux.'

(ParaSol)

À partir du XIX e siècle, pe de altă parte apparaît comme introducteur d'une information nouvelle, présentée parfois comme s'opposant à la conclusion du paragraphe précédent. L'exemple suivant illustre cet emploi de la locution pe de altă parte sans son antécédent initial pe de o parte. L'idée d'opposition y est confirmée par l'emploi de l'adverbe connecteur adversatif $d a r^{7}$ :

7. Mais on pourrait aussi dire, à l'inverse, que la présence de dar confirme que pe de altă parte tend à prendre un sens adversatif. 
[47] E neîndoielnic că fericirea în afara satisfacerii necesităților este greu de imaginat. Dar pe de altă parte, asta nu înseamnă că satisfacerea necesităților presupune neapărat fericirea. (Florin Mugur, Convorbiri cu Marin Preda, Bucarest, Editura Albatros, 1973, p. 224)

'Il est certain que le bonheur sans la satisfaction des nécessités est impossible. Mais, d'autre part, cela ne signifie pas que la satisfaction des nécessités apporte forcément le bonheur.'

(notre trad.)

Employé seul en tête de phrase et détaché du reste de l'énoncé, pe de altă parte (fr. «d'autre part», «en outre») peut fonctionner comme MD de changement de topique. Il exprime également un changement de perspective sur le sujet en question.

[48] Nu funcționează prea bine nici criteriile comparatiste, în schimb funcționează hipertrofierea valorilor altora prin supralicitare culturală. Pe de altă parte, critica face des, din superficialitate, confuzie între valoare și inovație, având tendinţa de a ratifica numai lucrul nou ca mod și formă [...].

(Gabriela Rusu-Păsărin, Interviul, recurs la memoria culturală, Bucarest, Tritonic Publishing, 2010, p. 195)

'Les critères comparatistes ne fonctionnent pas non plus, mais en revanche, l'hypertrophie des valeurs des autres fonctionne par surenchère culturelle. En outre, la critique littéraire fait souvent, par superficialité, la confusion entre valeur et innovation, ayant tendance à ratifier seule la nouveauté de forme et de manière.'

(notre trad.)

[49] Procuratura este una dintre instituţiile publice cel mai des acuzate în presa română. de cele mai multe ori acuzaţiile se referă la hotărîrile parchetului de netrimitere în judecată a unor persoane controversate şi la integritatea morală şi profesională a procurorilor. Pe de altă parte ziarele se vind mai bine şi audienţele cresc atunci cînd există cel puţin o rubrică de infracţiuni realizată aproape-ntotdeauna cu sprijinul procuraturii.

(IVLRA)

'Le parquet est l'une des institutions publiques qu'on accuse le plus souvent dans la presse roumaine. Dans la plupart des cas, les accusations visent les décisions de classement des dossiers de personnes controversés et l'intégrité des procureurs. D'autre part, les journaux se vendent bien et le public est plus intéressé quand il y a dans les journaux une rubrique consacrée aux infractions, réalisée presque toujours avec le soutien du parquet.'

(notre trad.)

[50] [...] deci din acest punct de vedere î: oricine poate vorbi despre Hagi fără nici un fel de reţineri şi pe de altă parte Hagi e şi un băiat echilibrat adică un om căruia nu i s-au urcat fumurile la cap cum spunea românul, care a păstrat şi o anumită modestie şi decenţă şi : seriozitate...

(IVLRA) 
[...] donc de ce point de vue n'importe qui peut parler de Hagi sans réserve et d'ailleurs Hagi est un garçon équilibré, c'est-à-dire un homme qui a gardé son sens de la réalité, comme on dit, qui a gardé aussi une certaine modestie et décence...'

(notre trad.)

\section{5. În altă ordine de idei (litt. dans un autre ordre d'idées)}

Cette périphrase - formée de la prép. $\hat{\imath} n+$ adj. indéf. altă + nom ordine + prép. de + nom idei et qui a comme équivalents en français les tournures par ailleurs, autre chose, d'autre part, d'un autre côté, dans un registre différent, comme deuxième sujet constitue un commentaire métadiscursif spécialisé pour signaler le changement radical de topique de discours. Sa fréquence en roumain contemporain nous laisse penser qu'il s'agit bien d'un marqueur en cours de pragmaticalisation.

[51] Întrucât relațiile transatlantice se bazează în primul rând pe un set de valori comune, sper că autoritățile americane vor fi mai receptive față de angajamentul UE de a renunța la pedeapsa cu moartea în întreaga lume. În altă ordine de idei, aș vrea să-i răspund colegului meu, domnul Preda. Faptul că avem această poziție legată cu abolirea pedepsei cu moartea nu înseamnă că subevaluăm parteneriatul strategic pe care îl avem cu Statele Unite ale Americii pe care ni-l dorim atât pentru Uniunea Europeană, cât și pentru țările noastre.

(https://www.linguee.com/)

'Puisque les relations transatlantiques reposent principalement sur un ensemble de valeurs communes, j'espère que les autorités américaines se montreront plus réceptives à l'engagement de l'UE à faire disparaître la peine de mort dans le monde entier. Dans un registre différent, je voudrais répondre à M. Preda. Le fait que nous ayons adopté cette position concernant l'abolition de la peine de mort ne signifie pas que nous sous-estimions le partenariat stratégique que nous avons avec les États-Unis et que nous souhaitons tant pour l'Union européenne que pour nos pays.'

(https://www.linguee.com/)

[52] Una din ideile legate de această taxă este faptul că ar putea trece neobservată, deoarece valoarea sa este de fiecare dată foarte redusă. În altă ordine de idei, sunt voci care sugerează utilizarea taxei pentru descurajarea tranzacţiilor excesive.

(https://www.linguee.com/)

'Une idée répandue, concernant la taxe, est que personne ne la remarquerait, car elle est très faible à chaque cas. Par ailleurs, d'autres suggèrent de l'utiliser pour dissuader les transactions excessives.'

(https://www.linguee.com/)

[53] Dar vă pot spune că NOPD va face tot posibilul să rezolve această situație. În altă ordine de idei, procurorii au renunţat la toate acuzaţiile împotriva profesorului 
Will Gerard, care a fost acuzat de uciderea reporterului de la postul din New Orleans, Alan Marsh.

(http://www.reverso.net/)

'Cependant, je peux vous dire que la police fera tout pour établir ce qui s'est passé. D'autre part, on apprend que le procureur a abandonné toutes les charges à l'encontre du professeur Will Gerard qui était accusé du meurtre d'Alan Marsh, journaliste au Post.'

(http://www.reverso.net/)

[54] În altă ordine de idei, aţi vorbit corect despre educaţie şi despre Procesul Bologna. (https://www.linguee.com/)

'D'un autre côté, vous avez parlé, et c'est bien, de l'enseignement et du processus de Bologne.'

(https://www.linguee.com/)

\subsection{Apropo (avec sa variante a propos) (du fr. «à propos»)}

Apropo est un adverbe emprunté au français au XIX ${ }^{e}$ siècle, ayant récemment acquis la valeur discursive de marqueur de digression, probablement sous l'influence du français. Comme marqueur de changement de topique, il peut signaler l'introduction d'un nouveau topique (exemple [55]) ou l'abandon d'un sujet en faveur d'un autre (exemple [57]) aussi bien que l'éloignement temporaire d'un topique récemment abordé, qui rappelle au sujet énonciateur un autre fait en relation ou non avec le topique respectif (exemples [56] et [58]).

[55] - Apropo - zice Mitică - de câţi ani ești ? - De... atâția. - Tocmai cât măgarul mitropolitului!

(Ion Luca Caragiale, Mitică, Bucarest, Adevărul, 1900)

'- À propos, quel âge as-tu? - J'ai... ans. - Tiens, tout comme lâne du cardinal!' (notre trad)

[56] Dând din mâini ca cel ce-alungă/ Gânduri rele: Eu sunt bun./ Apropo, ce-am vrut să spun?// Onofrei, te văd cu pungă,/ Dă-mi o mână de tutun.

(George Coşbuc, «Cetatea Neamţului», in Fire de tort, Bucarest, C. Sfetea, 1896)

'Et, tout en gesticulant comme quelqu'un qui cherche à chasser ses idées noires, il dit: «Je suis bon. $\grave{A}$ propos, qu'est-ce que je disais tout à l'heure? Onofrei, je vois que tu as un sac à tabac, donne m'en une poignée».'

(notre trad)

[57] Nimeni din familia mea nu se pricepe la magie, dar m-am bucurat foarte tare când am primit invitaţia pentru Hogwarts, e cea mai bună şcoală de vrăjitori! A propos, eu sunt Hermione Granger, voi?

(ParaSol) 
'Personne n'est sorcier dans ma famille, j'ai eu la surprise de ma vie en recevant ma lettre, mais j'étais tellement contente! On m'a dit que c'était la meilleure école de sorcellerie. Ah, au fait, je m'appelle Hermione Granger, et vous?'

(ParaSol)

[58] Thorache, întâmplător am dat de acest candid reprezentant al prieteniei între popoare. Apropo, văzusi cum îl cheamă ?

(https://www.garbo.ro/comunitate/forum/view_topic/8707/Cit-de-ridicol-eridicolul-pagina-16.html)

'Hé, mon Thor, c'est tout à fait par hasard que je suis tombé sur ce candide représentant de l'amitié entre les peuples. À propos, tu as vu son nom?'

(notre trad.)

\section{Bilan}

De l'analyse des différents emplois des lexèmes constituant le paradigme des MD de changement de topique du discours en roumain, il ressort que ces derniers se caractérisent: (i) par une sémantique propre fondée sur celle de l'unité lexicale qui en

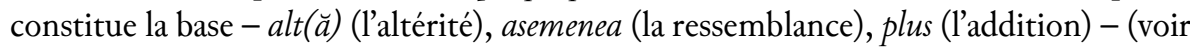
aussi Paillard et Franckel, 2008: 263); et (ii) par le fait qu'ils ont une distribution relevant d'une organisation généralement régulière.

Notre analyse de corpus a également mis en évidence les aspects suivants:

- les MD sont pour la plupart polyfonctionnels (la fonction de MD de changement de topique du discours étant le résultat d'un processus de grammaticalisation);

- ils servent à maintenir la cohérence du discours, tout en permettant de progresser dans le sujet;

- ils font partie des commentaires que le locuteur fait sur son activité énonciative et plus précisément sur l'architecture logique de son discours; de ce point de vue, ils sont proches des marqueurs de reformulation et des commentaires méta-énonciatifs;

- ils signalent des opérations cognitives que l'énonciateur verbalise afin de conserver la cohérence du discours, vue comme résultat de l'articulation logique des arguments, de la progression thématique et du respect des maximes de pertinence et de manière.

Du point de vue de leur grammaticalisation, nous avons identifié cinq grandes catégories de MD de changement de topique du discours en roumain, correspondant aux types de stratégies de marquage du changement de topique, qui expriment respectivement: 
1. une altérité dans la manière: (de) altfel, (de) altminteri;

2. une altérité spatiale: pe de altă parte;

3. une addition d'information: de asemenea, in plus, etc.;

4. un retour sur l'énonciation: apropo;

5. un commentaire sur le fait que l'énoncé hôte du MD, tout en s'inscrivant dans le prolongement des énoncés précédents, rompt avec leur contenu : în altă ordine de idei.

\section{Conclusion}

Dans cet article, nous avons analysé le parcours de pragmaticalisation des unités lexicales constituant le paradigme des MD de changement de topique en roumain, afin d'expliquer l'apparition des valeurs métadiscursives dans certains contextes d'emploi. À cet égard, on a vu d'abord que la constitution de ce paradigme de MD s'est finalisée vers le début du XIX ${ }^{\mathrm{e}}$ siècle, au moment où la langue roumaine a commencé un ample et complexe processus de modernisation. Cette affirmation est valable même pour les mots les plus anciens, hérités du latin, tels que (de) asemenea ou (de) altminteri, qui ont acquis le statut de MD beaucoup plus tard. Ce parcours évolutif montre aussi la tendance du roumain - qui s'observe d'ailleurs pour l'aire romane dans son ensemble - vers l'isomorphisme, c'est-à-dire vers une certaine correspondance entre forme et signification. Celle-ci a été illustrée par la pragmaticalisation de la paire: altfel - de altfel. Nous avons vu également que la pragmaticalisation des unités étudiées n'a pas complètement effacé leur sens initial et leurs valeurs d'origine.

En ce qui concerne le comportement fonctionnel des MD de changement de topique du discours en roumain, on remarque que le détachement en tête de phrase caractérise ces emplois récents des adverbes de manière, de quantité ou de lieu, la position initiale devenant même un critère de distinction de la fonction pragmatique assumée par ces unités lexicales (voir, à cet égard, de asemenea qui, pour remplir la fonction de marqueur de changement de topique, doit nécessairement se placer en tête de phrase). De même, nous avons vu que ces lexèmes inscrivent l'énoncé qui les héberge dans un enchaînement discursif et construisent un rapport étroit avec le contexte gauche. La position du MD de changement de topique dans l'énoncé, en lien avec le sémantisme propre du marqueur, peut signaler la continuité avec le topique antérieur (de altfel, de asemenea) ou bien la rupture énonciative (altfel, in altă ordine de idei).

Des recherches plus poussées devront, dans un premier temps, identifier et classer systématiquement toutes les expressions lexicalisées ayant pour fonction de signaler un changement de topique de discours en roumain contemporain. Dans un 
deuxième temps, il serait intéressant de comparer ${ }^{8}$ la classification des marqueurs de changement de topique du roumain avec celle de leurs correspondants dans les autres langues romanes, afin de voir quelles sont les convergences et les divergences dans l'origine et le type de grammaticalisation/pragmaticalisation des marqueurs assumant cette fonction dans les langues d'origine latine.

\section{Références bibliographiques}

Bazzanella, C. 1994. Le facce del parlare. Un approccio pragmatico all'italiano parlato. Florence: La Nuova Italia.

Charolles, M. 1995. Cohérence, cohésion et pertinence du discours. Travaux de linguistique 29: $125-151$.

Charolles, M. 2003. De la topicalité des adverbiaux détachés en tête de phrase. Travaux de linguistique $47:$ 11-49.

Chiorean, L. 2013. Despre conectorii textuali. Cu referire la adverb. In Proceedings of the "European Integration - Between Tradition and Modernity" Congress. Tîrgu-Mureş: Editura Universităţii „Petru Maior”. Vol. 5 : 295-305. En ligne à l'adresse suivante: http://www.diacronia.ro/indexing/details/A23378/pdf.

Chiorean, L. 2015. Conectorii textuali. Practica analizei instrucționale. In I. Pomian et N. Mocanu (éd.), Inspre şi dinspre Cluj. Contribuţii lingvistice. Omagiu profesorului G. G. Neamţu la 70 de ani. Cluj-Napoca: Argonaut: 124-135.

CHIRcu, A. 2012. Despre fel, „în multe chipuri”. Observații asupra lexicalizării și gramaticalizării adverbiale a unui substantiv, românescul fel. Limba română 61 (1) : 54-60. En ligne à l'adresse suivante: http://www.diacronia.ro/indexing/details/A478/pdf.

Combettes, B. 2003. Le topique comme constituant périphérique: aspects diachroniques. Travaux de linguistique 47: 137-161.

Combettes, B. 2007. Grammaticalisation des marqueurs de topicalisation en français: les expressions du type pour ce qui regarde. Langue française 156: 93-107.

Combettes, B. et PrÉvost, S. 2001. Évolution des marqueurs de topicalisation. Les cabiers de praxématique 37 : 103-124. En ligne à l'adresse suivante: https://journals.openedition. org/praxematique/230.

Croitor, B. 2015. Conectori transfrastici în limba română veche. In M. SALA, M. StANCiU Istrate et N. Petuhov (éd.), Lucrările celui de-al cincilea Simpozion Internațional de Lingvistică, București, 27-28 septembrie 2013 (SIL5). Bucarest: Univers Enciclopedic Gold: 235-251.

Dostie, G. 2004. Pragmaticalisation et marqueurs discursifs. Analyse sémantique et traitement lexicographique. Bruxelles: De Boeck-Duculot.

Ducrot, O. (éd.) 1980. Les mots du discours. Paris: Minuit.

8. La comparaison inter-langues des marqueurs de changement de topique du discours a été entamée dans le cadre du projet Labex TransferS 2014-2016 dirigé par Michel Charolles (http://www.lattice.cnrs.fr/ Operation-TransferS-Marqueurs-de-changement-de-topique-de-discours-Etudes). 
Fagard, B. et Charolles, M. 2018. Ailleurs, d'ailleurs, par ailleurs: de l'espace à l'humain, de l'humain au discours. Journal of French Language Studies 28 (3) : 351-375.

FAGARD, B. et SARDA, L. 2014. From Local Adverbials to Discourse Markers: Three Case Studies in the Diachrony of French. In L. SARDA, S. CARTER-Thomas, B. FAGARD et M. Charolles (éd.), Adverbials in Use. From Predicative to Discourse Functions. Louvain-la-Neuve: Presses universitaires de Louvain : 203-238.

Franckel, J.-J. et Paillard, D. 1997. Représentation formelle des mots du discours. Le cas de d'ailleurs. Revue de sémantique et de pragmatique $1: 51-64$.

Fraser, B. 1996. Pragmatic Markers. Pragmatics 6 (2): 167-190.

Fraser, B. 2009. Topic Orientation Markers. Journal of Pragmatics 41 (5) : 892-898.

Grice, H. P. 1975. Logic and Conversation. In J. P. Kimball, J. L. Morgan et P. Cole (éd.), Syntax and Semantics. New York - San Francisco - Londres: Academic Press. Vol. 3 : Speech Acts : 41-58.

Hansen, M.-B. M. et Rossari, C. (éd.) 2005. Journal of Historical Pragmatics: The Evolution of Pragmatic Markers 6 (2). Amsterdam - Philadelphie: J. Benjamins.

HoArță CăRăUșU, L. 2008. Dinamica morfosintaxei și pragmaticii limbii române actuale. Iași : Editura Cermi.

IONESCU, A. 2016. Étude comparative des évolutions sémantiques et pragmatiques des adverbes bien (en français) et bine (en roumain). Analele Universităţii din Craiova. Seria Ştiinţe Filologice. Lingvistică 38 (1-2): 195-215.

Lamiroy, B. et Charolles, M. 2004. Des adverbes aux connecteurs: simplement, seulement, malbeureusement, beureusement. Travaux de linguistique 49: 57-79.

LENK, U. 1998. Discourse Markers and Global Coherence in Conversation. Journal of Pragmatics 30 (2) : 245-257.

Marchello-Nizia, C. 2006. Grammaticalisation et changement linguistique. Bruxelles: De Boeck-Duculot.

MarChello-Nizia, C. 2007. Le principe de surprise annoncée: grammaticalisation et pragmaticalisation de cependant. Discours $1: 1-12$. En ligne à l'adresse suivante: https:// journals.openedition.org/discours/68.

MîRzeA VAsILE, C. 2012. Eterogenitatea adverbului românesc: tipologie și descriere. Bucarest : Editura Universității din București.

MongI, K. 2009. Autrement: un connecteur autrement polyfonctionnel. Langue française 161: 147-163.

Nordlinger, R. et Traugott, E. C. 1997. Scope and the Development of Epistemic Modality: Evidence from Ought to. English Language and Linguistics 1 (2): 295-317.

PaIllard, D. 2011. Marqueurs discursifs et scène énonciative. In S. Hancil (éd.), Marqueurs discursifs et subjectivité. Mont-Saint-Aignan: Presses universitaires de Rouen et du Havre: 13-39.

Paillard, D. et Franckel, J.-J. 2008. Mots du discours: adéquation et point de vue. L'exemple de réellement, en réalité, en effet, effectivement. Estudos linguisticos/Linguistic Studies 2: 255-274.

Pons Bordería, S. et Arguedas, M. E. 2009. Expressing Digression Linguistically: Do Digressive Markers Exist? Journal of Pragmatics 41 (5) : 921-936. 
Popescu, M. 2014. Romanian Atunci and French Alors: Functional and Discourse Properties. In C. Ghezzi et P. Molinelli (éd.), Discourse and Pragmatic Markers from Latin to the Romance Languages. Oxford - New York: Oxford University Press : 222-236.

Reboul, A. et Moeschler, J. 1998. Pragmatique du discours. De l'interprétation de l'énoncé à l'interprétation $d u$ discours. Paris: A. Colin.

Schwenter, S. A. et Traugott, E. C. 2000. Invoking Scalarity: The Development of In Fact. Journal of Historical Pragmatics 1 (1): 7-25.

ŞтEFănescu, A. 2007a. Aspecte pragmatice. Incursiuni în limba română actuală. Bucarest: Editura Universităţii din Bucureşti.

ŞтEFănescu, A. 2007b. Conectori pragmatici. Bucarest: Editura Universității din București.

SweEtser, E. 1990. From Etymology to Pragmatics: Metaphorical and Cultural Aspects of Semantic structure. Cambridge - New York - Melbourne: Cambridge University Press.

Traugott, E. C. 1982. From Propositional to Textual and Expressive Meanings: Some Semantic-Pragmatic Aspects of Grammaticalization. In W. P. Lehmann et Y. Malkiel (éd.), Perspectives on Historical Linguistics. Amsterdam - Philadelphie: J. Benjamins: 245-271.

Traugott, E. C. 1989. On the Rise of Epistemic Meanings in English: An Example of Subjectification in Semantic Change. Language 65 (1): 31-55.

Traugott, E. C. 1995. The Role of the Development of Discourse Markers in a Theory of Grammaticalization. In 12th International Conference on Historical Linguistics, Manchester, August 1995. 1-23. En ligne à l'adresse suivante: http://www.stanford.edu/ -traugott/ papers/discourse.pdf.

Traugott, E. C. et Dasher, R. B. 2002. Regularity in Semantic Change. Cambridge studies in linguistics 97. Cambridge: Cambridge University Press.

Van Dijk, T. A. 1977. Sentence Topic and Discourse Topic. Papers in Slavic Pbilology 1: 49-61. En ligne à l'adresse suivante: http://www.discourses.org/OldArticles/ Sentence\%20topic\%20and\%20discourse\%20topic.pdf.

\section{Corpus de référence: dictionnaires et bases de données}

Archeus.ro - Resurse lingvistice pentru limba română. En ligne à l'adresse suivante : http:// www.archeus.ro.

CDER - CIORăNescu, A. 2001 [1956-1966]. Dicţionarul etimologic al limbii române. Bucarest: Editura Saeculum.

CORV - DAscăLU-JINGA, L. 2002. Corpus de română vorbită (CORV). Eşantioane. Bucarest: Oscar Print.

DA - ACAdemia RomÂnă et Institutul DE Lingvistică „Iorgu Iordan - AleXANDru RosetTI" (éd.) 2010 [1913-1949]. Dicţionarul limbii române. Bucarest: Editura Academiei Române.

DEX - Coteanu, I., Seche, L., Seche, M. et Institutul De Lingvistică „Iorgu Iordan Alexandru Rosettı" (éd.) 2016. Dicţionarul explicativ al limbii române. Bucarest: Univers Enciclopedic Gold. 
DLR - ACADEmia RomÂnă et Institutul DE Lingvistică „IORgu IoRdAn - Alexandru RosettI” (éd.) 2010 [1958-2009]. Dicţionarul limbii române. Serie nouă. Bucarest: Editura Academiei Române.

DLRLC - ACADEMIA RoMÂNă (éd.) 1955-1957. Dicţionarul limbii române literare contemporane. Bucarest: Editura Academiei Republicii Populare Române.

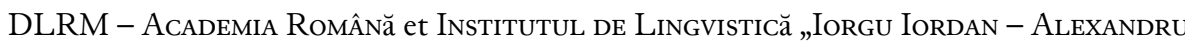
Rosettı" (éd.) 1958. Dicţionarul limbii române moderne. Bucarest: Editura Academiei Române.

GALR - Guţu Romalo, V. et Institutul de Lingvistică „Iorgu Iordan - Alexandru Rosettı” (éd.) 2005. Gramatica limbii române. Bucarest: Editura Academiei Române. Vol. II : Enunţul.

IVLRA - IONESCU-RuXăNDOIU, L. (éd.) 2002. Interacțiunea verbală în limba română actuală. Corpus (selectiv). Schiţă de tipologie. Bucarest: Editura Universităţii din Bucureşti.

ParaSol - A Parallel Corpus of Slavic and Other Languages. En ligne à l'adresse suivante : http://parasolcorpus.org/.

RDW - Tiktin, H. et Miron, P. 1986-1989. Rumänisch-Deutsches Wörterbuch. Wiesbaden : O. Harrassowitz. 